\title{
A GENERALIZATION OF HALL-COMPLEMENTATION IN FINITE SUPERSOLVABLE GROUPS
}

\author{
BY \\ HOMER BECHTELL
}

The purpose of this article is to characterize finite supersolvable groups $G$ satisfying one of the following properties:

$\mathscr{P}: G$ splits over each normal subgroup $N \$ \Phi(G), \Phi(G)$ the Frattini subgroup of $G$.

$\mathscr{P}^{*}$ : For each normal subgroup $N \$ \Phi(G)$, each reduced product of $G$ over $N$ is a semidirect product. $(G=N B$ is a reduced product over a normal subgroup $N$ by a subgroup $B$ iff $B$ does not contain a proper subgroup $B^{*}$ such that $G=N B^{*}$.)

F. Gross [5] has shown that for a finite solvable group $G$ having $\Phi(G)=1$, splitting over each normal subgroup is sufficient for the subgroup lattice to be complemented. As is known (e.g. see Theorem 24, [11]), this condition is sufficient for a supersolvable group $G$ to be Hall-complemented, i.e. for each subgroup $A$ of $G$ there is a subgroup $B$ of $G$ such that $G=A B=B A, A \cap B=1$, (see [7]). Furthermore, Hall-complementation is hereditary on the subgroups of $G$ and each reduced product is a semidirect product. So the groups $G$ satisfying either $\mathscr{P}$ or $\mathscr{P}^{*}$ whenever $\Phi(G)=1$ belong to the class of Hall-complemented groups. This article considers the case for $\Phi(G) \neq 1$.

Equivalently, if $G \in \mathscr{P}$, then for each short exact sequence $1 \rightarrow N \rightarrow G \rightarrow A \rightarrow 1$, such that $N \$ \Phi(G)$ and the epimorphism $\beta: G \rightarrow A$, there is associated a monomorphism $\tau: A \rightarrow G$, such that $\beta \tau=\mathrm{id}_{A}$. Also note that if $G \in \mathscr{P} *$ then $G \in \mathscr{P}$; in general the converse is not valid.

$\S \S 1$ and 2 give the basic properties and the preliminary results needed in order to identify the structure of the nonnilpotent supersolvable groups having property $\mathscr{P}$ given in $\S 3$. Among the results is that if $G \in \mathscr{P}$, then the Fitting subgroup $F(G) \in \mathscr{P}$. This necessitates the examination of the structure of the $p$-groups having these properties, and that is given in $\S \S 5$ and 6 . In $\S 4$ it is shown that if $F(G) \neq G$ is abelian, then $G \in \mathscr{P} *$ iff $G \in \mathscr{P}$. Also if $F(G) \neq G$ is nonabelian, $G \in \mathscr{P}$, and $F(G) \in \mathscr{P} *$, then $G \in \mathscr{P}^{*}$. Furthermore if $F(G)$ is a regular nonabelian $p$-group and $G \in \mathscr{P}$, then $G \in \mathscr{P} *$ iff $F(G) \in \mathscr{P} *$. Finally in $\S 7$ the structure is identified whenever $\mathscr{P}$ and $\mathscr{P} *$ are hereditary on $G$. In particular, $\mathscr{P}$ is hereditary on a $p$-group iff $\mathscr{P} *$ is hereditary on $G$ and the same result holds whenever $G$ is nonnilpotent.

Only finite supersolvable groups will be considered. Standard notation will be used throughout with clarification made whenever it is necessary.

Presented to the Society, January 24, 1969; received by the editors August 19, 1968. 
1. Elementary properties are given in the following propositions.

PROPOSITION 1.1. Each homomorphic image of a group $G \in \mathscr{P}$ (or $\mathscr{P} *)$ has property $\mathscr{P}($ or $\mathscr{P} *)$.

Proof. Let $N$ be a normal subgroup of $G$ containing the kernel $K$ of a homomorphism $\theta$ such that $N \theta \leqq \Phi(G \theta)$. Since $\Phi(G) \theta \leqq \Phi(G \theta)$, then $N \leqq \Phi(G)$ and $G=[N] A$ for some subgroup $A$ of $G$ (i.e. $G$ splits over $N$ by $A$ ). So $G / K=$ $[N / K](K A / K)$. Hence $G \theta \in \mathscr{P}$. Suppose $G \in \mathscr{P} *$ and $G / K=(N / K)(C / K)$ is a reduced product. Then $C$ contains a subgroup $C^{*}$ such that $G=[N] C^{*}$. Consequently $G / K=[N / K]\left(K C^{*} / K\right)$ and $K C^{*}=C$. Therefore $G \theta \in \mathscr{P} *$.

Proposition 1.2. For each homomorphism $\theta$ of a group $G \in \mathscr{P},(\Phi(G)) \theta=\Phi(G \theta)$.

Proof. Suppose $\Phi(G) \theta<\Phi(G \theta)$ properly. Then $G$ splitting over the inverse image of $\Phi(G \theta)$ contradicts $\Phi(G \theta) \neq 1$. Since $(\Phi(G)) \theta \leqq \Phi(G \theta)$, then equality results.

PROPOSITION 1.3. If $N$ is a normal subgroup of a group $G \in \mathscr{P}$, then either $N \leqq \Phi(G)$ or $\Phi(G)<N$.

Proof. If $N \cap \Phi(G) \neq N$ nor $\Phi(G)$, then $G=[N] C$ for some subgroup $C$ of $G$. For $\Phi(C) \neq 1, G=[N \Phi(C)] B$ and $|B|<|C|$, strictly. So under the natural homomorphism $\theta: G \rightarrow G / N \cong C, C \cong\langle\Phi(C), B\rangle \theta, \Phi(C) \theta=\Phi(C),|B \theta| \leqq|B|$, and $C \cong B \theta$. The contradiction implies $\Phi(C)=1$. Therefore $\Phi(G)<N$ or $N \leqq \Phi(G)$.

Corollary 1.3. If $G \in \mathscr{P}$ and $\Phi(G) \neq 1$, then the Fitting subgroup $F(G)$ is the Sylow p-subgroup for $p$ the largest prime dividing $|G|$.

Proof. If $G \in \mathscr{P}$ and $\Phi(G) \neq 1$, then $F(G)$ is a $p$-group by Theorem 1.3. That $F(G)$ is the Sylow $p$-subgroup for $p$ the largest prime dividing $|G|$ results from the supersolvability of $G$.

Proposition 1.4. For $A \neq 1, B \neq 1, G=A \otimes B \in \mathscr{P}$ iff $A$ and $B$ are Hall-complemented.

Proof. If $G \in \mathscr{P}$ then $\Phi(A)=\Phi(B)=\Phi(G)=1$ and $A$ and $B \in \mathscr{P}$. This implies that $A$ and $B$ are Hall-complemented. Conversely, if $A$ and $B$ are Hall-complemented, then $G$ is Hall-complemented, i.e. $G \in \mathscr{P}$ (see Theorem 24, [11]).

COROLlaRY 1.4.1. If $G \in \mathscr{P}$ then

(i) $Z(G) \leqq \Phi(G)$ or

(ii) $G=Z(G) \otimes A$, where the center of $G, Z(G)$, is a direct product of elementary abelian p-groups and $A$ is centerless and Hall-complemented.

COROLlaRY 1.4.2. If $G \in \mathscr{P}$ and $G$ is nilpotent then either

(i) $G$ is a nonabelian p-group or

(ii) $G$ is expressible as a direct product of elementary abelian groups. 
Assume that $G \in \mathscr{P}$ is not nilpotent and not expressible as a direct product with nontrivial factors. Consequently $1 \neq G^{\prime}=[G, G] \leqq F(G)$ and $\Phi(G)<G^{\prime}$ properly. Denote by $N$ a member of a principal series through $\Phi(G)$ for which $(\Phi(G): N)=p$ and consider $G^{*}=G / N$. Then $\Phi(G / N)=\Phi\left(G^{*}\right)<\left[G^{*}, G^{*}\right]$, properly, for otherwise $G$ would be nilpotent, and since $G^{*} \in \mathscr{P}, G^{*}$ is not expressible as a direct product, for this would contradict $\Phi\left(G^{*}\right) \neq 1$. Furthermore $\Phi\left(G^{*}\right)$ is the unique minimal normal subgroup. By Corollary $1.3, F(G)^{*}$ is a $p$-group, and of course $p \neq 2$, for then $G$ would be nilpotent.

Summarizing, one now has under consideration a group $G \in \mathscr{P}\left(G^{*}\right.$ in the above) having the following properties:

(1) $G$ is a nonnilpotent supersolvable group that is not expressible as a direct product,

(2) $F(G)$ is the Sylow $p$-subgroup for $p$ the largest prime dividing $|G|, p \neq 2$,

(3) $\Phi(G)<G^{\prime} \leqq F(G)$, and

(4) $\Phi(G)$ is the unique minimal normal subgroup of $G$.

For brevity, such a group will be called a $Q$-group.

2.

TheOREM 2.1. A Q-group $G \in \mathscr{P}$ iff $G$ satisfies one of the following conditions:

(i) $G=[F(G)] C$ for $F(G)$ cyclic of order $p^{2}, C$ cyclic of square-free order acting faithfully on $F(G)$, and $p \nmid|C|$.

(ii) $G=[F(G)] C$, where $F(G)$ is the nonabelian p-group of order $p^{3}$ and exponent $p$, and $C$ is the direct product of two cyclic subgroups of square-free order having at least one factor different from the identity, such that $C$ acts faithfully on $F(G), C$ fixes at least one maximal subgroup of $F(G)$ (and hence at least two), and $p \nmid|C|$.

Proof. Set $F(G)=F$. Clearly $\Phi(G)=\Phi(F)$. Since $\Phi(G)<G^{\prime}$ properly, there exists a subgroup $M \triangleleft G$ such that $\Phi(G)<M \leqq G^{\prime},|M|=p^{2}$, and $G=[M] A$ for $A$ a Hallcomplemented group. Hence there exists an elementary abelian $p$-group $A^{*}=F \cap A$ such that $F=[M] A^{*}$.

Case 1. $F^{\prime}=1$.

This immediately implies that $F=M \otimes A^{*}$ and so $M$ is cyclic of order $p^{2}$. Otherwise $\Phi(F)=1$ contradicts $\Phi(G) \neq 1$. If $A^{*} \neq 1$, then the elements of order $p$ in $F$ form a characteristic subgroup $H$ in $F$ and $(F: H)=p$. Since $G$ splits over $H$, this implies that $F$ is elementary abelian. Therefore $F=M$ is cyclic of order $p^{2}$. If $G=[F] C$, then $C$ is contained in Aut $(F)$ having $|C| \mid(p-1)$. Hence $C$ is a cyclic group of square-free order, $p \nmid|C|$.

Conversely if $G=[F] C$, where $F$ is cyclic of order $p^{2}$ for $p$ the largest prime dividing $|G|, C$ is cyclic of square-free order acting faithfully on $F$, and $p \nmid|C|$, one can readily verify that $G$ splits over each normal subgroup not contained in $\Phi(G)$.

Case 2. $F^{\prime}=\Phi(F)=\Phi(G) \neq 1$. 
Since $F^{\prime}<Z(F)$ properly implies that $F$ is abelian, then $F^{\prime}=Z(F)=\Phi(F)$. Moreover for the homomorphism $\theta: A^{*} \rightarrow \operatorname{Aut}(M)$, Ker $\theta \leqq Z(F) \leqq M$ implies that $\left|A^{*}\right|=p$. Hence $|F|=p^{3}$.

If $F$ does not have exponent $p$, then $F$ contains a subgroup $N$ of index $p$ generated by the elements of order $p$. Hence $N$ is characteristic in $F$ and normal in $G$. Consequently $G$ splits over $N$ but $F$ does not. Therefore $F$ is not of this type.

Suppose that $F$ has exponent $p$. Clearly $F$ will split over any subgroup, not $\Phi(F)$, that is normal in $G$ and contained in $F$. Since $F(G / \Phi(G))=F(G) / \Phi(G)$, then for $G^{*}=G / \Phi(G), F^{*}$ is elementary abelian of order $p^{2}$ and $F^{*}$ coincides with its centralizer. Moreover $G=[F] C$ implies that $G^{*}=[F / \Phi(G)](C \Phi(G) / \Phi(G)$ ), and so $G^{*}=\left[F^{*}\right] C^{*}$ where $C^{*} \cong C$. By Maschke's theorem, $F^{*}$ is completely reducible with respect to $C^{*}, C^{*}$ acting faithfully on $F^{*}$. However $G^{*}$ supersolvable implies that $F^{*}$ is the direct product of two subgroups of order $p$, say $F^{*}=F_{1}^{*} \otimes F_{2}^{*}$, such that $F_{1}^{*}, F_{2}^{*} \triangleleft G^{*}$. Then note that if $A^{*}$ is the kernel of the homomorphism of $C^{*} \rightarrow$ Aut $\left(F_{1}^{*}\right)$ and $A^{*} \neq 1$, no element different from the identity in $A^{*}$ can centralize $F_{2}^{*}$. So $C^{*}=A^{*} \otimes B^{*}$, where $B^{*} \neq 1, A^{*}$ and $B^{*}$ are cyclic of square-free order, $A^{*}$ centralizes $F_{1}^{*}$ and is faithful on $F_{2}^{*}$, whereas $B^{*}$ is faithful on $F_{1}^{*}$. Therefore $C^{*}$ is elementary abelian and no Sylow $q$-subgroup has order greater than $q^{2}$ for $q \neq p$.

Summarizing: If $F(G)$ has exponent $p$, then $G=[F] C$, where

(1) $C$ is faithful on $F$,

(2) $C$ is the direct product of two cyclic groups of square-free order, $A$ and $B$,

(3) $C$ fixes nontrivially two maximal subgroups $F_{1}$ and $F_{2}$ of $F$ such that $A$ is faithful on $F_{2}$ and $B$ is faithful on $F_{1}$,

(4) $p \nmid|C|, p$ odd.

Suppose that $G$ satisfies the conditions in the summary. Consider a subgroup $N \triangleleft G, N \neq 1$ nor $G$. Then $N \cap F=1$ implies that $N$ is contained in a subgroup conjugate to $C$ and consequently $N \leqq C$. Since $N \leqq Z(G)$, then $C$ is not faithful on $F$. So $N \cap F \neq 1$. There are then three cases to consider as follows:

Case A. $F \leqq N$.

Clearly $G$ splits over $N$.

Case B. $F \cap N \neq N, \neq F$.

There exists a self-normalizing maximal subgroup $L$ such that $N \$ L$. For if $N$ is contained in the intersection of all self-normalizing maximal subgroups of $G$, then by a result of Gaschütz [4], $N$ is nilpotent, and $N \leqq F$. Therefore $G=N L$. This implies the existence of a reduced product $N L^{*}=G$ such that $L^{*} \leqq L$ and $N \cap L^{*}$ $\leqq \Phi\left(L^{*}\right)$. However since $F(G) \$ L$, the Sylow $p$-subgroups of $L$, and hence $L^{*}$, are elementary abelian. By another result of Gaschütz (Satz 8 [3]), $L^{*}$ splits over each of its normal subgroups and so $\Phi\left(L^{*}\right)=1$. Therefore $G=[N] L^{*}$.

Case C. $N<F$.

Suppose $N \neq \Phi(G)=\Phi(F)$ and $|N|=p^{2}$. If $G^{\prime}=\Phi(F)$, then $G$ is nilpotent in contradiction to $C$ being faithful on $F$. Consequently there exists a self-normalizing 
maximal subgroup $L$ such that $N \$ L$ and $G=N L$. A reduced product exists, and as in Case B, $G$ splits over $N$.

Therefore the conditions in the summary are necessary and sufficient conditions that $G$ is a $Q$-group whenever $F^{\prime} \neq 1$.

REMARK. In the preceding theorem, the question arises that if $C$ fixes at least two maximal subgroups, does $C$ fix them all? In the group to be defined, $G$ is a $Q$-group belonging to $P$ and has precisely two maximal subgroups in $F(G)$ that are normal in $G$. Consider $G=\langle a, b, c, d\rangle$ with the defining relations $a^{7}=b^{7}=c^{7}=d^{3}=1, b^{-1} a b$ $=a c, a c=c a, b c=c b, d^{-1} a d=a^{2}, d^{-1} b d=b^{4}$, and $d^{-1} c d=c$. Then $F=F(G)=$ $\langle a, b, c\rangle, G=[F]\langle d\rangle, F=G^{\prime}$, and $\Phi(G)=\Phi(F)=F^{\prime}$.

3. Assume that $G$ is nonnilpotent, $G$ is not expressible as a direct product, and $\Phi(G) \neq 1$.

THEOREM 3.1. If $G \in \mathscr{P}$ then $F(G)$ is a p-group, $G=[F(G)] C, p \nmid|C|$, $p$ the largest prime dividing $|G|$, and $C$ is the direct product of two cyclic groups of square-free order, at least one of which differs from the identity.

Proof. The condition on $F(G)=F$ stems from Corollary 1.3. Since $G \in \mathscr{P}$, then $G=[F] C$ for some subgroup $C$ of $G$ and there exists a subgroup $N \triangleleft G$ such that $(\Phi(G): N)=p . G / N$ nilpotent implies that $G$ is nilpotent and if $G / N$ is expressible as a direct product then by Proposition 1.4, $\Phi(G) / N=1$. Therefore $G / N=$ $[F / N](C N / N)$ is a $Q$-group. The conditions on $C$ are the consequence of Theorem 2.1 together with the conjugacy of the complements of $F / N$.

THEOREM 3.2. If $G \in \mathscr{P}$, then $F(G)$ is abelian iff $F(G)$ is a cyclic p-group of order $p^{n}, n>1$.

Proof. By Corollary 1.3, $F(G)=F$ is a $p$-group, and, as in the proof of Theorem 3.1, there is a subgroup $N \triangleleft G$ such that $(\Phi(G): N)=p$ and $G / N$ is a $Q$-group. By Theorem 2.1(i), $F(G) / N=F(G / N)$ is cyclic. Hence $F$ is cyclic.

THEOREM 3.3. If $G \in \mathscr{P}$ then $F(G)=F$ is nonabelian iff

(i) $(F: \Phi(F))=p^{2}$,

(ii) $F$ is generated by two elements of order $p$,

(iii) $F$ splits over each $C$-invariant maximal subgroup, of which there are at least two, for the subgroup $C$ satisfying $G=[F] C$.

Proof. By Corollary 1.3, $F$ is a $p$-group, and, as in the above proof, there is a subgroup $N \triangleleft G$ such that $(\Phi(G): N)=p$ and $G / N$ is a $Q$-group. Then (i) and (iii) follow from Theorem 2.1(ii). Since there exists at least one maximal subgroup of $F$ that is normal in $G$, then the subgroup $H$ generated by the elements of order $p$ is not contained in $\Phi(F)$. Therefore $G$, and so $F$, splits over $H \Phi(F)$. However the complement of $H \Phi(F)$ in $F$ can have no elements of order $p$. Therefore $F=H \Phi(F)=H$. By (i), $F$ is a 2-generator group. So (ii) results.

If $G \in \mathscr{P}$ and the conditions are satisfied, then $F(G)$ can not be abelian. 
TheOREM 3.4. If $G \in \mathscr{P}$ and $F(G)=F$ is nonabelian, then $F \in \mathscr{P}$.

Proof. By Theorem 3.3, $(F: \Phi(F))=p^{2}$ and $F$ is generated by two elements $x, y$ of order $p$. Since no maximal subgroup of $F$ can contain both $x$ and $y$, then $F$ splits over each maximal subgroup. Furthermore if the subgroup $N \triangleleft F$ is not contained in $\Phi(F)$, then $F$ splits over $N \Phi(F)$ and so $N$ is maximal in $F$. Therefore $F \in \mathscr{P}$.

Corollary 3.4. If $G \in \mathscr{P}$, then $F(G) \in \mathscr{P}$.

Proof. By Theorem 3.2 if $F(G)$ is abelian, then $F(G)$ is cyclic of prime order and so $F(G) \in \mathscr{P}$, in a trivial way. On the other hand Theorem 3.4 takes care of the case for $F(G)$ nonabelian.

THEOREM 3.5. If $F(G)$ is abelian then $G \in \mathscr{P}$ iff for the largest prime $p|| G \mid$,

(i) $F(G)$ is cyclic of prime power order $p^{n}, n>1$, and

(ii) $G=[F(G)] C$, for $C$ a cyclic group of square-free order, $p \nmid|C|$, acting faithfully on $F(G)$.

Proof. If $G \in \mathscr{P}$ and $F(G)$ is abelian, then from our previous results, (i) and (ii) are valid. Assume that the conditions are satisfied and consider $N \triangleleft G, N\rfloor \Phi(G)$. Then $N$ splits over $F(N)=F \cap N$, say $N=[F(N)] H$, for some subgroup $H$ of $N$. Let $Q$ be a Sylow $q$-subgroup of $N, Q \leqq H, p \neq q$. Then $G=N \mathscr{N}(Q)$, where $\mathcal{N}(Q)$ is the normalizer of $Q$ in $G$. Clearly $\mathscr{N}(Q) \neq G$. Consider a Sylow $p$-subgroup $P$ of $\mathscr{N}(Q)$. It follows that $F(G)=F(N) P$. Since $F(G)$ is cyclic, then either $F(N)<P$ or $P=F(N)$. $P=F(N)$ implies that $F(G) \leqq N$ and so $G$ splits over $N$ since $((G: N),|N|)=1$. Otherwise, since $H$ is abelian, $N \leqq \mathscr{N}(Q)$. So $\mathscr{N}(Q)=G$, contrary to assumption.

THEOREM 3.6. If $F(G)$ is nonabelian then $G \in \mathscr{P}$ iff for the largest prime $p|| G \mid$

(i) $F(G)$ is a 2-generator p-group having property $\mathscr{P}$,

(ii) $G=[F(G)] C, p \nmid|C|$, where $C$ acts faithfully on $F(G)$ and $C$ is the direct product of two cyclic groups of square-free order, and

(iii) $G$ splits over each $C$-invariant maximal subgroup in $F(G)$, at least two of which exist.

Proof. The previous results yield the conditions if $G \in \mathscr{P}$. So assume that the conditions are satisfied. As in the proof of Theorem 3.5 consider $N \triangleleft G, N \Phi \Phi(G)$. Then $N=[F(N)] H$, for some subgroup $H$ of $N, F(N)=F(G) \cap N$. Denote by $Q$ a Sylow $q$-subgroup of $N, p \neq q, Q \leqq H$, for which $G=N \mathscr{N}(Q)$. Clearly $\mathscr{N}(Q) \neq G$. Suppose that $P$ is a Sylow $p$-subgroup of $\mathscr{N}(Q)$. Then $F(G)=F(N) P$. Since $F(G) \in \mathscr{P}$, then $F(N) \leqq \Phi(F(G))=\Phi(G)$ or $\Phi(G)<F(N)$. If $F(N) \leqq \Phi(G)$, then $F(G)=P$ and $\mathscr{N}(Q)=G$, contrary to our assumption. If $\Phi(G)<F(N)$ then either $F(N)=F(G)$ or else $F(N)$ is a $C$-invariant maximal subgroup of $F(G)$. In both cases $G=[F(N)] D$ such that the Sylow subgroups of $D$ are elementary abelian. By a result of Gaschütz (Satz 8, [3]), $D$ splits over each of its normal subgroups. Hence $N=[F(N)](N \cap D)$ and $D=[N \cap D] D^{*}$ yields $G=[N] D^{*}$. Therefore $G \in \mathscr{P}$. 
4. Assume that $G$ is nonnilpotent, that $G$ is not expressible as a direct product, and that $\Phi(G) \neq 1$.

THEOREM 4.1. If $F(G)$ is abelian then $G \in \mathscr{P} *$ iff $G \in \mathscr{P}$.

Proof. Consider $G \in \mathscr{P}$. By $\S 3, G=[F(G)] C$ is such that for the largest prime $p|| G \mid, F(G)$ is cyclic of order $p^{n}, n>1, C$ is cyclic of square-free order acting faithfully on $F(G)$, and $p \nmid|C|$. Consider a subgroup $N \triangleleft G$ such that $N \$ \Phi(G)=\Phi(F)$, form a reduced product $G=N D$, and assume $N \cap D \neq 1$. Then $N \cap D \leqq \Phi(D)$ and it is apparent that $\Phi(D)$, hence $N \cap D$, is a nontrivial p-group. Therefore $F(G)$ $=F(N) P$ for $F(N)=N \cap F(G)$ and $P$ the Sylow $p$-subgroup of $D$. If $F(G) \leqq N$ then $P=\Phi(P)=N \cap D$ implies $N \cap D=1$ and a contradiction. If $F(G) \$ N$ then $F(N)$ $\leqq \Phi(F(G))$ so that $F(G)=P$. Suppose that $y \in H$, for $N=[F(N)] H$, has order $q \neq p$, and $F(G)=\langle x\rangle$. Then $x^{-1}\langle y\rangle x=s^{-1}\langle y\rangle s$ for $s \in F(N)=\left\langle x^{p^{m}}\right\rangle$, i.e. $s=x^{k p^{m}}$. So $\langle y\rangle=\left(x^{k p^{m}+1}\right)^{-1}\langle y\rangle\left(x^{k p^{m}+1}\right)$ and hence $F(G) \leqq N\langle y\rangle$. Therefore $\langle y\rangle \triangleleft N F(G)$ and $[\langle y\rangle, F(G)] \leqq\langle y\rangle \cap F(G)=1$. Since $y$ centralizes $F(G)$ then $y \in F(G)$, a contradiction. Therefore $N \cap D=1$ and each reduced product of $G$ over $N$ is a semidirect product.

THeOREM 4.2. If $G \in \mathscr{P}$ and $F(G)$ is nonabelian, then $F(G) \in \mathscr{P} *$ implies that $G \in \mathscr{P} *$.

Proof. Suppose $N \triangleleft G$ and consider $N \cap F(G)$. Since $F=F(G) \in \mathscr{P}$ then either $N \cap F \leqq \Phi(F)=\Phi(G)$ or $\Phi(G)<N \cap F$ properly. If $N \cap F \leqq \Phi(G)$ then $\Phi(N)$ $\leqq N \cap F$. Hence $N$ is nilpotent and contained in $F$. So either $N \leqq \Phi(G)$ or $\Phi(G)<N$, properly. Consider a reduced product $G=N H$ and assume $F \leqq N$. Then $N \cap H$ $=\Phi(H)$ since $\Phi(H)$ must be a $p$-group. However $p \nmid|G| N \mid$ and so $H$ splits over $N \cap H$. Thus $N \cap H=1$. Then suppose that $\Phi(G)<N \cap F<F$ properly. Again $\Phi(H)=N \cap H$. Since $N \cap F$ is a maximal subgroup of $F$, then the index of $\Phi(H)$ in the Sylow $p$-subgroup of $H$ is $p$, and $F(H)$ is cyclic. However if $F \in \mathscr{P} *$ and $x \in F \mid \Phi(F)$, then $x$ must be of prime order. Otherwise $F$ contains a maximal subgroup $M$ such that $x \notin M$ and $F=M\langle x\rangle$. If $\left.|\langle x\rangle|=p^{n}, n\right\rangle 1$, then $F$ has a reduced product that is not a splitting product. Therefore $n=1$. Hence $\Phi(H)=N \cap H=1$, i.e. the reduced product is a semidirect product.

COROLlaRY 4.2. If $G \in \mathscr{P}$ and $F(G)$ is a regular nonabelian p-group, then $G \in \mathscr{P} *$ iff $F(G) \in \mathscr{P} *$.

Proof. If $G \in \mathscr{P} *$ then $F(G) \in \mathscr{P}$ by Theorem 3.4. Since $F(G)$ is a regular nonabelian $p$-group generated by two elements of order $p$, then $F(G)$ has exponent $p$. Hence $F(G) \in \mathscr{P} *$. Theorem 4.2 gives the converse.

5. Since each nilpotent group $G$ is the direct product of its Sylow subgroups, then by Proposition 1.4 if $G \in \mathscr{P}$ and $|G|$ is divisible by two distinct primes, then $G$ is a direct product of elementary abelian subgroups. The same can be said about an abelian $p$-group expressible as a direct summand with at least two factors differing 
from the identity. However for completeness in this case, if $G$ is cyclic of order $p^{n}$, $n>1$, then $G \in \mathscr{P}$. Consequently for the remainder of this section it will be assumed that $G \in \mathscr{P}$, that $G$ is not expressible as a direct product, and that $G$ is nonabelian. From $\S 1$ it immediately follows that

(1) $Z(G) \leqq \Phi(G)$,

(2) for the subgroup $N \triangleleft G$, either $N \leqq \Phi(G)$ or $\Phi(G)<N$, and

(3) each homomorphic image of $G$ has property $\mathscr{P}$.

THEOREM 5.1. If $G \in \mathscr{P}$ then $\Phi(G)=G^{\prime}$.

Proof. If $G \in \mathscr{P}$ then $G / G^{\prime} \in \mathscr{P}$ and hence is elementary abelian or cyclic. $G / G^{\prime}$ cyclic implies that $G$ is cyclic and therefore a contradiction.

THEOREM 5.2. $G \in \mathscr{P}$ iff $G$ is generated by two elements of order $p$.

Proof. It will be enough to show that $(G: \Phi(G))=p^{2}$. Denote by $N$ a subgroup normal in $G$ such that $(\Phi(G): N)=p$ and consider $G_{1}=G / N$. Then $G_{1}^{\prime}=G^{\prime} \mid N$ $=\Phi\left(G_{1}\right)=\Phi(G) / N$. Since $G_{1} \in \mathscr{P}$ is nonabelian then $Z\left(G_{1}\right)=\Phi\left(G_{1}\right)$. So there exists a normal subgroup $N_{1}$ of $G_{1}$ such that $\left(N_{1}: \Phi(G)\right)=p$. Hence $G_{1}=\left[N_{1}\right] P_{1}$. Under conjugation by elements of $P_{1}$ there is a homomorphism of $P_{1}$ into Aut $\left(N_{1}\right)$. Since the kernel is in $Z\left(G_{1}\right)$, it follows from $\left|N_{1}\right|=p^{2}$, that $\left|P_{1}\right|=p$. Therefore $\left(G_{1}: \Phi\left(G_{1}\right)\right)$ $=(G: \Phi(G))=p^{2}$. Since $G$ is nonabelian and $G \in \mathscr{P}$, the two generating elements of order $p$ exist.

For the converse note that $G^{\prime}$ must be $\Phi(G)$. If $N$ is a subgroup normal in $G$, then $N \Phi(G)$ is a maximal subgroup of $G$. Hence $N$ must be a maximal subgroup. Consequently each normal subgroup of $G$ is either in $\Phi(G)$ or contains $\Phi(G)$. Therefore $G \in \mathscr{P}$.

Corollary 5.2.1. If $G \in \mathscr{P}$, then there exists a G-normal subgroup $N,(\Phi(G): N)$ $=p$, such that $G_{1}=G / N$ is nonabelian,

(i) $\left|G_{1}\right|=p^{3}$,

(ii) for $p$ odd, $G_{1}$ has exponent $p$, and

(iii) for $p=2, G_{1}$ is the dihedral group.

COROLlaRY 5.2.2. For $p=2, G \in \mathscr{P}$ iff $G$ is a dihedral group.

Proof. As is known, the only nonabelian 2-groups generated by two elements of order 2 are the dihedral groups. The converse is readily verified.

REMARK. As the result of Corollary 5.2.2, the remaining discussion will consider only $p$-groups for $p \neq 2$.

THEOREM 5.3. A nonabelian p-group $G$ having at least two abelian maximal subgroups has property $\mathscr{P}$ iff $G$ is the nonabelian group of order $p^{3}$ having exponent $p$.

Proof. If nonabelian $G \in \mathscr{P}$ and has two abelian maximal subgroups, then $G^{\prime}=\Phi(G)=Z(G)$. Therefore each maximal subgroup of $G$ is abelian and hence each proper subgroup of $G$ is abelian. Groups with this property have $\left|G^{\prime}\right|=p$. So $|G|$ $=p^{3}$ and it follows that $G$ has exponent $p$. The converse is evident. 
It can be noted that if $G \in \mathscr{P}$ and has precisely one abelian maximal subgroup then $G$ has maximal class. This stems from $\left(G: G^{\prime}\right)=p^{2}$. Another observation is that if $G \in \mathscr{P}$ and it is a nonabelian $p$-group then $G$ cannot be the Frattini subgroup of any p-group. This follows from a result of C. Hobby [9], namely: A nonabelian $p$-group $G$, the index of whose derived group is $p^{2}$, cannot be $\Phi\left(G^{*}\right)$ for any $p$-group $G^{*}$. Also if $G \in \mathscr{P}$ and $|G|=p^{4}, p$ odd, then $G$ satisfies one of the following types:

(i) $G=\left\langle x, y, z, w^{\prime}\right| x^{p}=y^{p}=z^{p}=w^{p}=1, w^{-1} z w^{\prime}=z x, z^{-1} y z=y, z^{-1} x z=x, w^{-1} x w^{\prime}$ $\left.=x, z^{-1} x z=x, y^{-1} x y=x\right\rangle$, for $p>3$,

(ii) $G=\left\langle x, y, z \mid x^{9}=y^{3}=z^{3}=1, y^{-1} x y=x, z^{-1} x z=x y, z^{-1} y z=x^{-3} y\right\rangle$.

6. Consider $G \in \mathscr{P} *$ and assume that $G$ is a nonabelian p-group that is not expressible as a direct product.

THEOREM 6.1. $G \in \mathscr{P} *$ iff

(i) $G \in \mathscr{P}$ and

(ii) $\Phi(G)$ contains all elements of order $p^{n}, n>1$.

Proof. Consider $x \in G \backslash \Phi(G), G \in \mathscr{P} *$. There exists a maximal subgroup $M$ such that $x \notin M$ and $G=M\langle x\rangle$. Since each reduced product must be a semidirect product, it follows that $|x|=p$. The converse is clear.

COROllaRy 6.1.1. $G \in \mathscr{P} *$ iff $G$ is a 2-generator group having each element in $G \backslash \Phi(G)$ of order $p$.

COROLlaRy 6.1.2. If $G \in \mathscr{P}^{*}$ and $1=Z_{0}<\cdots<Z_{n}=G$ is the ascending central series of $G$, then $Z_{i} / Z_{i-1}$ is an elementary abelian group.

Proof. If $x \in Z(G)$ has order $p^{n}$, for $n>1$, then each $y \in G \backslash \Phi(G)$ has the same order in contradiction to the theorem.

In the following a regular p-group refers to that defined by P. Hall [6] (or see [10]).

THEOREM 6.2. If $G \in \mathscr{P}$ then $G$ is regular iff each element of $G$ has order $p$.

Proof. If each element has order $p$ then $G$ is regular. On the other hand for $G$ regular, $G_{p}$ the subgroup generated by the elements of order $p$, and $G^{p}$ the subgroup generated by the $p$ th power of the elements of $G,\left|G / G_{p}\right|=\left|G^{p}\right|$, (e.g. see Satz 10.7, p. 327, [10]). Since $G=G_{p}$ then $G$ has exponent $p$.

COROllary 6.2. If $G$ is regular then $G \in \mathscr{P}$ iff $G \in \mathscr{P} *$.

THEOREM 6.3. The only nonabelian 3-group $G$ that belongs to $\mathscr{P} *$ has exponent 3 and order $3^{3}$.

Proof. Let $\Phi^{2}(G)=\Phi(\Phi(G))$. Then by Theorem $6.1, G / \Phi^{2}(G)$ has each element of order 3 and hence it is a regular 3-group with two generators. By Satz 10.3, p. 322, [10], such a group has $G / \Phi^{2}(G)$ cyclic. However since $G^{\prime}=\Phi(G)$, then $G^{\prime} / \Phi^{2}(G)$ cyclic implies that $G^{\prime}$ is cyclic. Suppose that $|G| \geqq 3^{4}$. Then $G$ contains a normal subgroup $N<G^{\prime}$ such that $|G / N|=3^{4}$. However, as noted in the remarks at the end 
of $\S 5$, there are no nonabelian 3 -groups of this order that satisfy $\mathscr{P} *$ and have a cyclic commutator subgroup. Therefore $|G|<3^{4}$.

Corollary 6.3. If $G \in \mathscr{P}^{*}$ and $|G|>p^{3}$, then $p>3$.

Proof. Only the dihedral groups belong to $\mathscr{P}$ for $p=2$.

REMARK. For the remaining portion of this section assume that $p>3$.

THEOREM 6.4. If $G \in \mathscr{P} *$ then either

(i) $G$ has exponent $p$ or

(ii) $\left(G: G^{p}\right) \geqq p^{p}, G^{p} \neq 1$.

Proof. Consider $G / G^{p}$ which is regular by Theorem 6.2 and satisfies $\mathscr{P} *$. Denote $p^{\omega}=\left|G / G^{p}\right|$. P. Hall [8] has shown that whenever $\omega<p$, then $G$ is regular. This implies that $\left|G^{p}\right|=1$. Therefore $\omega \geqq p$.

THEOREM 6.5. Let $G \in \mathscr{P}^{*}$ and $|G|=p^{n}, p>3$. Then each element in $\Phi(G)$ has order $p^{r}$ for $r<n-p+1$ whenever $n>p$.

Proof. Suppose that $\Phi(G)$ has an element of order $p^{n-p+1}$. A result of $\mathrm{N}$. Blackburn (Corollary 2.2, [2]) is that either $\left|G / G^{p}\right|<p^{p}$ or $G$ possesses a normal subgroup $N$ of order $p^{p}$ and exponent $p$ such that $G / N$ is cyclic, or $G$ is a $p$-group of maximal class of order $p^{p+1} .\left|G / G^{p}\right|<p^{p}$ implies that $G$ is regular and has exponent p. $G / N$ cyclic implies that $G^{\prime}=\Phi(G)<N$ and so each element of $G$ has order $p$. A known result (see p. 369, [10]) is that if $G$ has maximal class with $|G|=p^{n}, 5 \leqq n \leqq p$ +1 then $G^{\prime}$ has exponent $p$. However another known result (see p. 373, [10]) is that if $G$ has maximal class with $|G| \geqq p^{p+1}$ then $G$ is irregular. Combining the last two statements with Theorem 6.2 one has that $|G|$ cannot be $p^{p+1}$ whenever $G$ has maximal class. Hence $\Phi(G)$ cannot have elements $x$ such that $|x|=p^{r}$ for $r \geqq n$ $-p+1$.

COROLlaRY 6.5.1. If $G \in \mathscr{P *}$ then the series $1=G_{k}<G_{k-1}<\cdots<G_{1}<G$, where $G_{k-j}$ is the subgroup generated by the $p^{k-j}$ powers of the elements of $G$, has $k<n$ $-p-1$.

Proof. By Theorem 6.5, $k<n-p+1$, and since the subgroup of $\Phi(G)$ that is generated by the $p^{i}$ powers of the elements coincides with that for $G$ then $k<(n-2)$ $-p+1=n-p-1$.

COROLlaRy 6.5.2. Let $G \in \mathscr{P} *$. If $G$ is of maximal class, then $|G| \leqq p^{p}$.

Proof. Examine the proof of the theorem.

THEOREM 6.6. Let $G \in \mathscr{P} *$. If $|G|=p^{n}$ for $n \geqq 5, p>3$, then

(i) $\left(\Phi(G): \Phi^{2}(G)\right) \geqq p^{3}$ and

(ii) $\Phi(G)$ contains at least one G-normal elementary abelian subgroup of order $p^{3}$.

Proof. A result of Blackburn (see p. 16, [2]) is that if $|G|=p^{n}, p$ odd, $n \geqq 5$, and all normal subgroups of $G$ of order $p^{r}$ have two generators for fixed $r, 3 \leqq r \leqq n-2$, 
then either $G$ is metacyclic, or $G$ is a 3-group of maximal class, or for $r=3, G$ contains a nonabelian normal subgroup $N$ of exponent $p$ such that $|N|=p^{3}$ and $G / N$ is cyclic. Suppose $G \in \mathscr{P}^{*}$ and $\left(\Phi(G): \Phi^{2}(G)\right)=p^{2}$. Since there exists only one normal subgroup of order $p^{n-2}$, namely $\Phi(G)$, Blackburn's result is applicable. Clearly $G$ cannot be metacyclic and Theorem 6.3 takes care of the case for $p=3$. The last case implies $|G|=p^{4}$. Consequently $\left(\Phi(G): \Phi^{2}(G)\right) \neq p^{2} . \Phi(G)$ cannot be cyclic since this would imply the existence of a nonabelian group of order $p^{4}$ satisfying $\mathscr{P}$ with cyclic Frattini subgroup, a contradiction. Therefore

$$
\left(\Phi(G): \Phi^{2}(G)\right) \geqq p^{3} .
$$

As for (ii) note that each normal subgroup of order $p^{3}$ is contained in $\Phi(G)$. If none of them are elementary abelian, then all are 2-generator groups. Applying the same result of Blackburn's, a contradiction is reached and the result follows.

THEOREM 6.7. Let $G \in \mathscr{P}^{*}$. If $G$ is irregular, then $G$ contains a normal subgroup $N$ such that

(i) $N<\Phi(G)$ (properly),

(ii) $|N| \geqq p^{p-1}$,

(iii) $N$ has exponent $p$, and

(iv) $|G|>p^{p+1}$.

Proof. If $|G|=p^{p+1}$ and $G$ is irregular, then $G$ has class $p$, (see p. 331, [10]). This contradicts Corollary 6.5.2. So $|G|>p^{p+1}$. If $G$ has no normal subgroup $N$ of exponent $p$ with $|N| \geqq p^{p-1}$ then $G$ is regular (see p. 334, [10]). So (ii) and (iii) are satisfied. Since $\Phi(G) \leqq N$ implies that $G$ is regular, then $N<\Phi(G)$ properly.

7.

THEOREM 7.1. $\mathscr{P}$ is hereditary on a 2-group $G$ iff $G$ is either

(i) elementary abelian or

(ii) a dihedral group.

Proof. (i) is evident and if $G$ is nonabelian then by Corollary 5.2.2, $G$ is a dihedral group. As can be readily verified, each subgroup of type (ii) satisfies $\mathscr{P}$.

THEOREM 7.2. $\mathscr{P}$ is hereditary on a p-group $G$ for an odd prime $p$ iff $\mathscr{P} *$ is hereditary on $G$.

Proof. Assume that $\mathscr{P}$ is hereditary on $G$. A result of Blackburn (Theorem 4, [1]) is that if $G$ and $G^{\prime}$ are generated by two elements, then $G^{\prime}$ is abelian. So $\Phi(G)$ must be abelian and hence it is either elementary abelian or it is cyclic of prime order, $p^{n}$. If $n>1$ it cannot be cyclic since the only group of order $p^{4}$ satisfying $\mathscr{P}$ has $\Phi(G)$ noncyclic. If $n=1$, then $G$ is nonabelian of order $p^{3}$ and has exponent $p$, i.e. $\mathscr{P} *$ is hereditary on $G$.

Assume that $\Phi(G)$ is elementary abelian of order $p^{n}, n>1$, and use induction on $|G|$. Each abelian maximal subgroup of $G$ is elementary abelian and inductively each 
nonabelian maximal subgroup has exponent $p$. Hence $G$ has exponent $p$ and $\mathscr{P} *$ is hereditary on $G$.

The converse is evident.

THEOREM 7.3. $\mathscr{P} *$ is hereditary on a p-group $G$ iff $G$ satisfies one of the following:

(i) $G$ is cyclic, or

(ii) $G$ is elementary abelian, or

(iii) $G$ is nonabelian, $p>2$, and

(a) $|G| \leqq p^{p}$,

(b) $G$ has maximal class, and

(c) $G=[M]\left\langle x \mid x^{p}=1\right\rangle$ for $M$ an elementary abelian p-group.

Proof. Assume that $\mathscr{P} *$ is hereditary on $G$. Note that $G$ would be elementary whenever $p=2$ and Theorem 6.3 completes the case for $p=3$. So assume $p>3, G$ is nonabelian, and $|G|>p^{3}$. For a subgroup $N \triangleleft G$ of index $p^{4}$, the centralizer $M / N$ of $G^{\prime} \mid N$ has index $p$ and so it is elementary abelian. Therefore $M$ is abelian and $G$ has maximal class. By Corollary 6.5.2, $|G| \leqq p^{p}$.

For the converse it is enough to examine (iii). Since $|G| \leqq p^{p}, G$ is regular, and by Corollary 6.2, $G \in \mathscr{P} *$. Each abelian subgroup of $G$ is elementary abelian. If $G$ contains a nonabelian maximal subgroup $K$, then $K \in \mathscr{P}$ and so $K \in \mathscr{P} *$. Inductively each nonabelian subgroup satisfies $\mathscr{P}^{*}$.

THEOREM 7.4. For a nonnilpotent supersolvable group $G, \mathscr{P}$ is hereditary on $G$ iff $\mathscr{P}^{*}$ is hereditary on $G$.

Proof. First note that $F(G)$ is not a 2 -group by Theorem 3.1. Then $\mathscr{P}$ hereditary on $G$ implies that $\mathscr{P}$ is hereditary on $F(G)$. By Theorem $7.2, \mathscr{P}^{*}$ is hereditary on $F(G)$ and so $F(G)$ must satisfy one of the forms in Theorem 7.3. By Theorem 4.2, $G \in \mathscr{P} *$. However this is valid for all nonnilpotent subgroups of $G$. So $\mathscr{P} *$ is hereditary on $G$.

THEOREM 7.5. $\mathscr{P} *$ is hereditary on a nonnilpotent supersolvable group $G$ iff $G$ satisfies one of the following:

(i) $G$ is Hall-complemented or

(ii) $G=[F] C$ where for the largest prime $p$ dividing $|G|, F$ is a p-group, $p \nmid|C|, C$ acts faithfully on $F$,

(a) $F$ is cyclic of order $p^{n}, n>1$ and $C$ is a cyclic group of square-free order or

(b) $F$ is nonabelian of maximal class, $|F| \leqq p^{p}, F=[M]\left\langle x \mid x^{p}=1\right\rangle$ for $M$ an elementary abelian p-group, $C$ is a direct product of two cyclic groups of square-free order, at least one of which differs from the identity, and either

(1) $|F|=p^{3}$ and $F$ has exponent $p$ or

(2) $|F|>p^{3}$ and $C$ is faithful on each $C$-invariant maximal subgroup of $F$.

Proof. Since $\mathscr{P} *$ is hereditary on $G$ then $F(G)=F \in \mathscr{P} *$. Then (i) arises whenever $F(G)$ is elementary abelian, (iia) is a consequence of Theorem 3.5 and the general 
form of (iib) is the result of Theorems 3.6 and 7.3. In the case of (iib), if $F(G)$ has two abelian maximal subgroups then $|F|=p^{3}$ and $F(G)$ has exponent $p$ by Theorem 5.3. Otherwise if $F(G)$ has precisely one abelian maximal subgroup and $N$ is a nonabelian maximal subgroup of $F(G)$ invariant under $C$, then $[N] C$ exists in $G$. If $C_{1}<C$ centralizes $N$, then $[N] C=[N] C_{2} \otimes C_{1}$, for $C_{2}$ such that $C=C_{1} \otimes C_{2}$. $\mathscr{P} *$ hereditary on $G$ implies that $[N] C_{2}$ is Hall-complemented by Corollary 1.4.1. Thus $N$ is an elementary abelian $p$-group, a contradiction. Therefore $C$ is faithful on each $C$-invariant maximal subgroup in $F$. This completes (iib).

In the introduction it was pointed out that $\mathscr{P} *$ is hereditary on $G$ whenever $G$ was Hall-complemented. So assume that (iia) is satisfied. By Theorems 3.5 and 4.1, $G \in \mathscr{P} *$. Since $p \nmid|C|$, then $C$ acting faithfully on $F$ implies that $C$ acts faithfully on each subgroup of $F$ and hence this permits induction on $|G|$. If $N$ is a normal maximal subgroup of $G$, then $F \leqq N$ and $N=[F] C_{1}$ where $C_{1}$ is conjugate to a subgroup of $C$. Therefore $C_{1}$ is faithful on $F$ and inductively $\mathscr{P} *$ is hereditary on $N$. On the other hand if $S$ is a self-normalizing maximal subgroup of $G$, then $S$ $=[S \cap F] C^{*}$ where $C^{*}$ is a conjugate of $C$. As noted $C^{*}$ is faithful on $S \cap F$, and again inductively $\mathscr{P} *$ is hereditary on $S$. Since $N$ and $S$ were arbitrary and $G \in \mathscr{P} *$ it follows that $\mathscr{P} *$ is hereditary on $G$.

Next assume that the general structure in (iib) is satisfied. By Theorem $7.3, \mathscr{P} *$ is hereditary on $F$ and from Theorems 3.6 and $4.2, G \in \mathscr{P} *$, for both (1) and (2). If $N$ is a maximal subgroup of $G$ and $F<N$, then $\mathscr{P} *$ is hereditary on $N$ by induction. For case (1), if $N$ is a maximal subgroup of $G$ such that $F+N$ then $N \cap F$ is elementary abelian, $(G: N)=p$, and hence all Sylow subgroups of $N$ are elementary abelian. By a result of Gaschütz (see Satz 8, [3]) $N$ splits over each normal subgroup. Since $N$ is supersolvable it follows that $N$ is Hall-complemented (see introduction). Therefore $\mathscr{P} *$ is hereditary on each subgroup of $G$.

For case (2) of (iib), note that since $F$ has maximal class, then $|F|>p^{3}$ implies that $F$ possesses precisely one abelian maximal subgroup, namely $M$, and hence $M \triangleleft G$. If $N$ is a maximal subgroup of $G$ and $F \leqq N$, it follows by induction that $\mathscr{P} *$ is hereditary on $N$. If $F \$ N$, then $(G: N)=p$ since $G$ is supersolvable. $M<N$ implies that $N$ splits over each normal subgroup by the previously mentioned result of Gaschütz. Hence $N$ is Hall-complemented. Otherwise $(F \cap N) \neq M,(F \cap N) \triangleleft N$, and $(F \cap N)$ is a nonabelian maximal subgroup of $F$. Then note that $N=[F \cap N] C^{*}$ where $C^{*}$ is a conjugate of $C$. By hypothesis $C^{*}$ is faithful on $F \cap N$ and either $|F \cap N|=p^{3}$ and $F \cap N$ has exponent $p$ or else $F \cap N$ satisfies (2). By induction, $\mathscr{P}^{*}$ is hereditary on $N$. Consequently $\mathscr{P}^{*}$ is hereditary on $G$.

8. It should be noted that if we were to generalize Hall-complementation by requiring that for each subgroup $A$ of a group $G, A \nsubseteq \Phi(G)$, there is a subgroup $B$ such that $G=A B=B A, A \cap B \leqq \Phi(G)$, then the class is enlarged considerably. For example, all nonabelian $p$-groups having $(G: \Phi(G))=p^{2}$ would be included.

One of the motivations for the discussion of groups satisfying either $\mathscr{P}$ or $\mathscr{P} *$, 
in particular for $p$-groups, rests in the determination of a structure that would enable a $p$-group to be isomorphic to the Frattini subgroup of some finite group. It has not been determined whether or not this is dependent upon the group structure with respect to the reduced products over the normal subgroups not contained in the Frattini subgroup. If it is, then the natural question would be, does a strong group property such as introduced in this paper yield an equally strong structural condition on $\Phi(G)$ ? Also it raises the question on the type of structure that a supersolvable group must possess in order that it does not split over any proper normal subgroup and the resulting effect on the structure of the Frattini subgroup.

ACKNOWLEDGEMENT. This work was partially supported by NSF Grant GP7130. I appreciate the comments and the suggestions of the referee.

\section{BIBLIOGRAPHY}

1. N. Blackburn, On prime-power groups in which the derived group has two generators, Proc. Cambridge Philos. Soc. 53 (1957), 19-27.

2. - Generalization of certain elementary theorems on p-groups, Proc. London Math. Soc. 11 (1961), 1-22.

3. W. Gaschütz, Zur Erweiterungstheorie der endlicher Gruppen, J. Reine Angew. Math. 190 (1952), 93-107.

4. — Uber die $\Phi$-Untergruppe endlicher Gruppen, Math. Z. 58 (1953), 160-170.

5.. F. Gross, Uberauflosbare Gruppen mit elementar-abelschen Sylowgruppen, Ph.D. Thesis, Christian-Albrechts Universitat, Kiel, 1962.

6. P. Hall, A contribution to the theory of groups of prime power orders, Proc. London Math. Soc 36 (1933), 29-95.

7. — Complemented groups, J. London Math. Soc. 12 (1937), 201-204.

8. - The classification of prime-power groups, J. Reine Angew. Math. 182 (1940), $130-141$.

9. C. Hobby, The Frattini subgroup of a p-group, Pacific J. Math. 10 (1960), 209-212.

10. B. Huppert, Endliche Gruppen. I, Springer-Verlag, Berlin, 1967.

11. M. Suzuki, Structure of a group and the structure of its lattice of subgroups, Ergebnisse der Math. und ihrer Grenzgebiete, vol. 10, Springer-Verlag, Berlin, 1956.

UNIVERSITY OF NEW HAMPSHIRE, Durham, NeW HAMPSHIRE 